\title{
Multicenter Analysis of Clinical Features and Prognosis of COVID-19 Patients with Hepatic Impairment
}

\author{
Jeong Eun Song ${ }^{1}$, Min Kyu Kang ${ }^{2}$, Yu Rim Lee ${ }^{3}$, Chang Hyeong Lee ${ }^{1}$, Jung Gil Park², Young Oh Kweon ${ }^{3}$, Won \\ Young Tak ${ }^{3}$, Soo Young Park ${ }^{3}$, Se Young Jang ${ }^{3}$, Jae Seok Hwang ${ }^{4}$, Byoung Kuk Jang ${ }^{4}$, Won Young Jang ${ }^{4}$, Jeong \\ III Suh ${ }^{5}$, Woo Jin Chung ${ }^{3}$, and Byung Seok Kim¹, Daegu-Gyeongbuk Liver Study Group (DGLSG) \\ ${ }^{1}$ Department of Internal Medicine, Daegu Catholic University School of Medicine, ${ }^{2}$ Department of Internal Medicine, Yeungnam \\ University College of Medicine, ${ }^{3}$ Department of Internal Medicine, School of Medicine, Kyungpook National University, ${ }^{4}$ Department \\ of Internal Medicine, Keimyung University School of Medicine, Daegu, and ${ }^{5}$ Department of Internal Medicine, Dongguk University \\ College of Medicine, Gyeongju, Korea
}

\section{Article Info}

Received August 22, 2020

Revised January 5, 2021

Accepted January 6, 2021

Published online April 2, 2021

\section{Corresponding Author}

Byung Seok Kim

ORCID https://orcid.org/0000-0002-4318-4570

E-mail kbs9225@cu.ac.kr

Woo Jin Chung

ORCID https://orcid.org/0000-0002-3736-1067

E-mail chung50@dsmc.or.kr
Background/Aims: Recent data indicate the presence of liver enzyme abnormalities in patients with coronavirus disease 2019 (COVID-19). We aimed to evaluate the clinical features and treatment outcomes of COVID-19 patients with abnormal liver enzymes.

Methods: We performed a retrospective, multicenter study of 874 COVID-19 patients admitted to five tertiary hospitals from February 20 to April 14, 2020. Data on clinical features, laboratory parameters, medications, and treatment outcomes were collected until April 30, 2020, and compared between patients with normal and abnormal aminotransferases.

Results: Abnormal aminotransferase levels were observed in 362 patients (41.1\%), of which 94 out of $130(72.3 \%)$ and 268 out of $744(36.0 \%)$ belonged to the severe and non-severe COVID-19 categories, respectively. The odds ratios (95\% confidence interval) for male patients, patients with a higher body mass index, patients with severe COVID-19 status, and patients with lower platelet counts were 1.500 (1.029 to $2.184, p=0.035), 1.097(1.012$ to $1.189, p=0.024)$, 2.377 ( 1.458 to $3.875, p=0.001$ ), and 0.995 ( 0.993 to $0.998, p>0.001)$, respectively, indicating an independent association of these variables with elevated aminotransferase levels. Lopinavir/ ritonavir and antibiotic use increased the odds ratio of abnormal aminotransferase levels after admission (1.832 and 2.646, respectively, both $p<0.05)$. The median time to release from quarantine was longer ( 22 days vs 26 days, $p=0.001)$ and the mortality rate was higher $(13.0 \%$ vs $2.9 \%$, $p<0.001)$ in patients with abnormal aminotransferase levels.

Conclusions: Abnormal aminotransferase levels are common in COVID-19 patients and are associated with poor clinical outcomes. Multivariate analysis of patients with normal aminotransferase levels on admission showed that the use of lopinavir/ritonavir and antibiotics was associated with abnormal aminotransferase levels; thus, careful monitoring is needed. (Gut Liver 2021;15:606-615)

Key Words: SARS-CoV-2; COVID-19; Aminotransferase; Prognosis; Lopinavir-ritonavir

\section{INTRODUCTION}

An outbreak of coronavirus disease 2019 (COVID-19) caused by severe acute respiratory syndrome coronavirus 2 (SARS-CoV-2) began in December 2019 from Wuhan, China, and spread rapidly across the world. On March 11, 2020, the World Health Organization declared the COV-
ID-19 pandemic. By April 11, 2020, more than 1.6 million patients were diagnosed with COVID-19, with 100,000 deaths reported worldwide. ${ }^{1}$ COVID-19 is currently an ongoing public health emergency of international concern.

The clinical course of COVID-19 ranges from asymptomatic infection to severe progressive pneumonia and death. ${ }^{2}$ Common clinical manifestations of COVID-19 
include dry cough, fever, fatigue, and other signs of respiratory tract infection. ${ }^{3,4}$ Although SARS-CoV-2 is known to cause respiratory tract infections, several large-scale hospital-based studies have reported that $14 \%$ to $53 \%$ of COVID-19 patients present with elevated levels of alanine aminotransferase (ALT) and aspartate aminotransferase (AST). ${ }^{3-6}$ A recent study reported abundant SARS-CoV-2 viral particles in hepatocytes of severe COVID-19 patients, suggesting that SARS-CoV-2 induces this liver damage. Drug-related hepatotoxicity may also induce liver enzyme abnormalities. Since there is no defined treatment regimen for COVID-19, antibiotics and antiviral agents are widely used. These drugs may cause liver damage while undergoing therapy. However, data on the association between liver enzyme abnormalities and drug use are limited.

In this study, we evaluated hepatic parameters in COVID-19 patients admitted to five tertiary hospitals in Daegu, South Korea, and compared the clinical features, medications, and clinical outcomes between the COVID-19 patients with and without elevated aminotransferase.

\section{MATERIALS AND METHODS}

\section{Patients and study design}

Medical records of patients diagnosed with COVID-19 at five tertiary hospitals in Daegu, South Korea from February 17, 2020 to April 6, 2020 were reviewed. The diagnosis and discharge of COVID-19 patients were performed according to guidelines issued by the Korea Disease Control and Prevention Agency. ${ }^{8}$ All cases were diagnosed based on SARS-CoV-2 RNA detection by real-time polymerase chain reaction from nasopharyngeal and oropharyngeal swabs. Affected patients were discharged if they met both clinical criteria (absence of fever and improved clinical symptoms) and testing criteria (two consecutive negative results on polymerase chain reaction tests within a 24-hour interval).

Patients with more than two laboratory examinations within 2 weeks of hospitalization were enrolled in the study. To evaluate clinical features and treatment outcomes in COVID-19 patients with abnormal liver enzymes, enrolled patients were classified into two groups: normal aminotransferase and abnormal aminotransferase. Abnormal aminotransferase was defined as ALT or AST levels higher than the upper limit of normal (ULN) values in two consecutive laboratory tests conducted every 3 to 7 days. The ULN values of AST and ALT were defined as $34 \mathrm{U} / \mathrm{L}$ in males and $30 \mathrm{U} / \mathrm{L}$ in females.

This study was performed according to the ethical guidelines of the revised Helsinki Declaration of 2013. The study protocol was reviewed and approved by the institutional review board of all tertiary hospitals (IRB number: CR-20-094). Documentation of informed consent from study participants was exempted as this study was a retrospective analysis of existing clinical data.

\section{Data collection}

We retrospectively collected medical records, including epidemiological variables, clinical characteristics, laboratory and radiological findings, and treatment and outcome data for each patient. All data were collected until April 30, 2020. Two researchers independently reviewed the data. Laboratory data included complete blood cell counts, Creactive protein, erythrocyte sedimentation rate, biochemical tests, liver function tests, and kidney function tests on the day of admission to the hospital. To define the abnormal liver enzymes group, results of liver function tests, repeated every 3 to 7 days, were collected. For each patient, the peak values of ALT, AST, and other hepatic parameters were selected for analysis. Chest radiograph findings of patients on admission were retrospectively reviewed. A chest radiograph with a patch shadow, interstitial changes, or consolidation was defined as abnormal.

\section{Definitions}

According to the World Health Organization interim guidance on COVID-19, severe patients were diagnosed based on the presence of any of the following characteristics: (1) respiratory distress, respiration rate $\geq 30$ times/ minute; (2) oxygen saturation at resting state $\leq 93 \%$; and (3) partial pressure of oxygen/fraction of inspired oxygen $\leq 300 \mathrm{~mm} \mathrm{Hg}$ by arterial blood gas analysis. ${ }^{10}$ Systemic inflammatory response syndrome (SIRS) on admission was defined by the presence of any two of the following conditions: (1) body temperature $<36^{\circ} \mathrm{C}$ or $>38^{\circ} \mathrm{C}$, (2) heart rate $>90$ beats/minute, (3) tachypnea $>20$ breaths/minute, and (4) white blood cell counts $<400 / \mu \mathrm{L}$ or $>12,000 / \mu \mathrm{L}$. The definition of shock and acute respiratory distress syndrome (ARDS) was based on the World Health Organization interim guidance. ${ }^{10}$ Acute kidney injury was defined as the highest serum creatinine level $(>0.3 \mathrm{mg} / \mathrm{dL}$ within 48 hours or 1.5 times of the baseline level within 7 days) and/ or decreased urine output $(<0.6 \mathrm{ml} / \mathrm{kg} / \mathrm{hr}$ for 6 hours).

\section{Treatment strategies}

There are currently no standardized guidelines for drug choice or accepted antiviral regimens for the treatment of COVID-19 patients. Patients were treated with lopinavir/ ritonavir, darunavir/cobicistat, or hydroxychloroquine on the day of admission based on physicians' discretion. Antibiotics were administered if necessary. Oxygen support, 
including nasal cannula, high-flow nasal cannula, and invasive mechanical ventilation, was provided based on the severity of hypoxemia.

\section{Statistical analysis}

Continuous variables were described as the median and interquartile range, and categorical variables as frequencies and percentages. The means for continuous variables were compared using the Student t-test or the Mann-Whitney $\mathrm{U}$ test. Comparison of categorical variables was performed using the chi-square test or Fischer exact test. Multivariable logistic regression analysis was conducted to explore the risk factors for abnormal liver enzymes in COVID-19 patients. p-values $<0.05$ were considered statistically significant. SPSS version 25.0 (IBM Corp., Armonk, NY, USA) was used for all statistical analyses.

\section{RESULTS}

\section{Clinical characteristics}

In total, 1,005 patients were admitted to five tertiary hospitals from February 17, 2020 to April 6, 2020. We excluded 131 patients due to a lack of laboratory tests during hospitalization. Of the remaining 874 eligible patients, $362(41.4 \%)$ and $512(58.6 \%)$ patients were categorized into abnormal and normal aminotransferase groups, respectively. The number of patients experienced elevated aminotransferase at least once during hospitalization is summarized in Supplementary Table 1. Baseline characteristics, clinical features, and treatment regimens of patients in both groups are summarized in Table 1 . The median age in both groups was 62 years, with no significant difference between the groups. The ratio of males was higher in patients with abnormal liver enzymes than in patients with normal liver enzymes ( $47.2 \%$ vs $26.8 \%, \mathrm{p}<0.001)$. With the exception of a higher proportion of patients with coronary artery disease in the abnormal liver enzyme group (6.2\% vs $2.4 \%, \mathrm{p}=0.014$ ), no significant differences in comorbidities between the groups were noted.

Forty-four patients $(5.0 \%)$ had chronic liver disease, including 30 with non-cirrhotic disease, seven with compensated cirrhosis, and seven with decompensated cirrhosis. The most common etiology of chronic liver disease was chronic hepatitis B $(n=21,2.4 \%)$, followed by chronic hepatitis $C(n=10,1.1 \%)$, alcoholic liver disease $(n=9,1.0 \%)$, and autoimmune hepatitis $(\mathrm{n}=1,0.1 \%)$. No significant differences were observed in underlying liver disease between the groups.

Although the prevalence of initial symptoms was similar in both groups, patients with abnormal liver enzymes presented with a higher proportion of fever $(52.2 \%$ vs $39.9 \%, \mathrm{p}=0.001)$ and dyspnea ( $34.3 \%$ vs $19.6 \%, \mathrm{p}<0.001)$. The median time from onset of symptoms to hospitalization was 6 days, with no significant difference between the groups. Patients with SIRS and severe COVID-19 on admission were more common in the abnormal aminotransferase group (SIRS: $21.5 \%$ vs $7.3 \%, \mathrm{p}<0.001$; severe COVID-19: $26.0 \%$ vs $7.0 \%, \mathrm{p}<0.001)$. Ninety-four of 130 (72.3\%) severe COVID-19 patients and 268 of 744 (36.0\%) non-severe COVID-19 patients presented with abnormal aminotransferases. Abnormal chest radiographs (70.4\% vs $53.9 \%, \mathrm{p}<0.001)$ and bilateral involvement $(55.0 \%$ vs $33.0 \%, \mathrm{p}<0.001)$ were observed more frequently in the abnormal aminotransferase group. More patients with abnormal liver enzymes received lopinavir/ritonavir $(64.9 \%$ vs $50.0 \%, \mathrm{p}<0.001)$, darunavir/cobicistat $(6.9 \%$ vs $3.5 \%$, $\mathrm{p}=0.022)$, hydroxychloroquine ( $63.0 \%$ vs $48.4 \%, \mathrm{p}<0.001)$, and antibiotic ( $87.6 \%$ vs $70.1 \%, \mathrm{p}<0.001)$ treatment.

The laboratory results of both groups are summarized in Table 2. Most patients with abnormal liver enzymes exhibited elevation of ALT (48.3\%) and AST (50.3\%) of one to two times the ULN. Levels of alkaline phosphatase (ALP) and $\gamma$-glutamyl transferase were higher in patients with abnormal liver enzymes than in patients with normal liver enzymes, even though all median values were within the normal ranges. Liver function abnormalities, including lower albumin ( $42.3 \%$ vs $19.0 \%, \mathrm{p}<0.001)$, higher total bilirubin $(20.9 \%$ vs $8.7 \%, \mathrm{p}<0.001)$, and higher international normalized ratio $(11.1 \%$ vs $4.8 \%, \mathrm{p}=0.001)$ were more frequent in patients with abnormal liver enzymes than in those with normal liver enzymes. Patients with abnormal liver enzymes presented with higher white blood cell counts $(5,450 / \mu \mathrm{L}$ vs $5,195 / \mu \mathrm{L}, \mathrm{p}=0.001)$ and lower platelet counts $\left(200 \times 10^{3} / \mu \mathrm{L}\right.$ vs $\left.231 \times 10^{3} / \mu \mathrm{L}, \mathrm{p}<0.001\right)$, although median values were within the normal ranges. C-reactive protein levels were higher in the abnormal liver enzyme group than in the normal group $(3.8 \mathrm{mg} / \mathrm{L}$ vs $0.8 \mathrm{mg} / \mathrm{L}$, $\mathrm{p}=0.008$ ). Biochemical parameters were comparable between the groups.

\section{Factors associated with abnormal liver enzymes in COVID-19 patients}

Among 362 patients with abnormal liver enzymes, 158 had peak levels of aminotransferase higher than twice the ULN. We subsequently examined the clinical factors associated with elevation of more than twice the ULN in COVID-19 patients (Supplementary Table 2). The univariate and multivariate logistic regression analyses (Table 3) showed that male sex (odds ratio [OR], 1.500; 95\% confidence interval [CI], 1.029 to $2.184 ; \mathrm{p}=0.035$ ), higher body mass index (OR, 1.097; 95\% CI, 1.012 to 1.189 ; $\mathrm{p}=0.024$ ), 
Table 1. Clinical Characteristics of Patients with COVID-19 According to Liver Test Results

\begin{tabular}{|c|c|c|c|c|}
\hline \multirow{2}{*}{ Characteristics } & \multicolumn{2}{|c|}{ Liver tests (aminotransferase) } & \multirow{2}{*}{ Total } & \multirow{2}{*}{ p-value } \\
\hline & Normal & Abnormal & & \\
\hline No. of patients & $512(58.6)$ & $362(41.4)$ & 874 & \\
\hline Age, yr & $62(49-73)$ & 62 (52-73) & $62(51-73)$ & 0.707 \\
\hline Male sex & $137(26.8)$ & $171(47.2)$ & $308(35.2)$ & $<0.001$ \\
\hline Body mass index, $\mathrm{kg} / \mathrm{m}^{2}$ & $23.2(20.9-25.0)$ & $24.7(22.3-26.7)$ & $23.7(21.7-26.0)$ & 0.337 \\
\hline \multicolumn{5}{|l|}{ Comorbidity } \\
\hline Diabetes & 105 (20.5) & 75 (20.7) & $180(20.6)$ & 0.940 \\
\hline Hypertension & $168(32.8)$ & $115(31.8)$ & $283(32.4)$ & 0.745 \\
\hline Coronary artery disease & $13(2.4)$ & $21(6.2)$ & 34 (3.9) & 0.014 \\
\hline Chronic obstructive pulmonary disease & $6(1.2)$ & $7(1.9)$ & $13(1.5)$ & 0.359 \\
\hline Chronic kidney disease & $12(2.3)$ & $4(1.1)$ & $16(1.8)$ & 0.178 \\
\hline \multicolumn{5}{|l|}{ Liver-related disease } \\
\hline \multicolumn{5}{|l|}{ Etiology } \\
\hline Chronic hepatitis B & $12(2.3)$ & $9(2.5)$ & $21(2.4)$ & 0.892 \\
\hline Chronic hepatitis C & $8(1.6)$ & $2(0.6)$ & $10(1.1)$ & 0.167 \\
\hline Alcoholic liver disease & $4(0.8)$ & $5(1.4)$ & $9(1.0)$ & 0.387 \\
\hline Autoimmune hepatitis & 0 & $1(0.3)$ & $1(0.1)$ & 0.414 \\
\hline Others & 0 & $3(0.8)$ & $3(0.3)$ & 0.710 \\
\hline \multicolumn{5}{|l|}{ Liver cirrhosis } \\
\hline No cirrhosis & $17(3.3)$ & $13(3.6)$ & $30(3.4)$ & 0.828 \\
\hline Compensated cirrhosis & $3(0.6)$ & $4(1.1)$ & $7(0.8)$ & 0.396 \\
\hline Decompensated cirrhosis & $3(0.6)$ & $4(1.1)$ & $7(0.8)$ & 0.457 \\
\hline \multicolumn{5}{|l|}{ Signs and symptoms on admission } \\
\hline Fever/chills & 201 (39.9) & 189 (52.2) & $390(45.0)$ & 0.001 \\
\hline Cough & $283(56.3)$ & $218(60.4)$ & $501(58.0)$ & 0.226 \\
\hline Dyspnea & $99(19.6)$ & 124 (34.3) & $223(25.8)$ & $<0.001$ \\
\hline Gastrointestinal symptoms (nausea/vomiting/diarrhea) & $114(22.6)$ & $90(24.9)$ & $204(23.6)$ & 0.443 \\
\hline Myalgia & $161(31.9)$ & 127 (35.2) & 288 (33.3) & 0.319 \\
\hline Headache & $139(27.6)$ & $85(23.5)$ & 224 (25.9) & 0.182 \\
\hline Days from symptom onset to admission & $6(3-9)$ & $6(3-8)$ & $6(3-9)$ & 0.565 \\
\hline SIRS on admission & $37(7.3)$ & $78(21.5)$ & 115 (13.2) & $<0.001$ \\
\hline Severe patients on admission & $36(7.0)$ & $94(26.0)$ & $130(14.9)$ & $<0.001$ \\
\hline \multicolumn{5}{|l|}{ Radiologic findings on admission } \\
\hline Abnormal chest radiographs & $276(53.9)$ & $255(70.4)$ & $531(60.8)$ & $<0.001$ \\
\hline Bilateral involvement of chest radiographs & $169(33.0)$ & $199(55.0)$ & $368(42.1)$ & $<0.001$ \\
\hline \multicolumn{5}{|l|}{ Treatment } \\
\hline Lopinavir/ritonavir & $256(50.0)$ & 235 (64.9) & $491(56.2)$ & $<0.001$ \\
\hline Darunavir/cobicistat & $18(3.5)$ & $25(6.9)$ & $43(4.9)$ & 0.022 \\
\hline Hydroxychloroquine & $248(48.4)$ & $228(63.0)$ & $476(54.5)$ & $<0.001$ \\
\hline Antibiotics & $359(70.1)$ & $317(87.6)$ & $676(77.3)$ & $<0.001$ \\
\hline
\end{tabular}

Data are presented as the number (\%) or median (interquartile range).

COVID-19, coronavirus disease 2019; SIRS, systemic inflammatory response syndrome.

severe COVID-19 on admission (OR, 2.377; 95\% CI, 1.458 to $3.875 ; \mathrm{p}=0.001$ ), and lower platelet count (OR, 0.995; $95 \%$ CI, 0.993 to 0.998 ; $\mathrm{p}<0.001$ ) were independently associated with elevated aminotransferase levels.

To evaluate the effects of drugs on abnormal aminotransferase, subgroup analysis of the 550 patients with normal aminotransferase levels on admission was performed. Among these patients, 130 (23.6\%) presented with abnormal aminotransferase levels during hospitalization, predominantly elevation of AST and ALT levels by one to three times the ULN ( $\mathrm{n}=101,77.7 \%)$. Baseline characteristics, clinical features, and treatment regimens according to abnormal liver enzymes after admission are summarized in Supplementary Table 3. Multivariate logistic regression was used to adjust for age, sex, underlying liver disease, COVID-19 severity, SIRS, and radiographic findings on admission. Among drugs potentially linked to elevated aminotransferase, including lopinavir/ritonavir, darunavir/ cobicistat, hydroxychloroquine, and antibiotics, the use of lopinavir/ritonavir (OR, 1.832; 95\% CI, 1.155 to 2.919; $\mathrm{p}=0.011$ ) and antibiotics (OR, 2.646; 95\% CI, 1.452 to 4.824; $\mathrm{p}<0.001$ ) was associated with a higher risk of abnormal liver enzymes (Fig. 1). 
Table 2. Laboratory Findings of Patients with COVID-19 According to Liver Test Results

\begin{tabular}{|c|c|c|c|c|}
\hline \multirow{2}{*}{ Characteristics } & \multicolumn{2}{|c|}{ Liver tests (aminotransferase) } & \multirow{2}{*}{ Total } & \multirow{2}{*}{ p-value } \\
\hline & Normal & Abnormal & & \\
\hline No. of patients & $512(58.6)$ & $362(41.4)$ & 874 & \\
\hline \multicolumn{5}{|c|}{ Peak values of liver test results during hospitalization } \\
\hline \multicolumn{5}{|c|}{ Liver enzymes } \\
\hline ALT, U/L & $18(14-24)$ & $51(37-79)$ & $25(17-45)$ & $<0.001$ \\
\hline Normal & $512(100)$ & $58(16.0)$ & $570(65.2)$ & $<0.001$ \\
\hline $1-2$ ULN & 0 & $175(48.3)$ & $175(20.0)$ & \\
\hline$>2$ ULN & 0 & $129(35.6)$ & $129(14.6)$ & \\
\hline AST, U/L & $19(16-23)$ & $44(34-70)$ & $24(18-39)$ & 0.005 \\
\hline Normal & $512(100)$ & $72(19.9)$ & $584(66.8)$ & $<0.001$ \\
\hline $1-2$ ULN & 0 & 182 (50.3) & $182(20.8)$ & \\
\hline$>2$ ULN & 0 & $108(29.8)$ & $108(12.4)$ & \\
\hline$A L P, U / L$ & $62(49-75)$ & $75(60-97)$ & 67 (53-83) & $<0.001$ \\
\hline$\leq 130$ & $471(97.5)$ & $311(89.1)$ & $782(94.0)$ & $<0.001$ \\
\hline$>130$ & $12(2.5)$ & $38(10.9)$ & $50(6.0)$ & \\
\hline GGT, U/L & $19(13-32)$ & $36(22-76)$ & $23(15-46)$ & $<0.001$ \\
\hline$\leq 40$ & 109 (85.2) & $49(52.1)$ & $158(71.2)$ & $<0.001$ \\
\hline$>40$ & $19(14.8)$ & 45 (47.9) & $64(28.8)$ & \\
\hline \multicolumn{5}{|l|}{ Liver functions } \\
\hline Total bilirubin, mg/dL & $0.5(0.4-0.7)$ & $0.7(0.4-1.1)$ & $0.6(0.4-0.9)$ & $<0.001$ \\
\hline$\leq 1.2$ & 397 (91.3) & $268(79.1)$ & 665 (85.9) & $<0.001$ \\
\hline$>1.2$ & $38(8.7)$ & $71(20.9)$ & $109(14.1)$ & \\
\hline Serum albumin, g/dL & $4.0(3.6-4.2)$ & $3.6(3.0-4.1)$ & $3.9(3.4-4.2)$ & $<0.001$ \\
\hline$<3.5$ & $97(19.0)$ & $153(42.3)$ & $250(28.6)$ & $<0.001$ \\
\hline$\geq 3.5$ & $414(81.0)$ & 209 (57.7) & $623(71.4)$ & \\
\hline Prothrombin time, INR & $1.0(0.9-1.0)$ & $1.0(1.0-1.1)$ & $1.0(0.9-1.1)$ & 0.005 \\
\hline$\leq 1.2$ & 394 (95.2) & 297 (88.9) & 665 (92.5) & 0.001 \\
\hline$>1.2$ & $20(4.8)$ & $34(11.1)$ & 54 (7.5) & \\
\hline \multicolumn{5}{|l|}{ On admission } \\
\hline \multicolumn{5}{|l|}{ Complete blood count } \\
\hline White blood cell count, $/ \mu \mathrm{L}$ & $5,195(4,022-6,568)$ & $5,450(4,218-7,240)$ & $5,300(4,077-6,735)$ & 0.001 \\
\hline Lymphocyte count, $/ \mu \mathrm{L}$ & $1,430(1,052-1,857)$ & $1217(807-1,718)$ & $1,350(960-1,810)$ & 0.080 \\
\hline Platelet count, $\times 10^{3} / \mu \mathrm{L}$ & $231(179-290)$ & $200(157-263)$ & $216(166-279)$ & $<0.001$ \\
\hline Hemoglobin, g/dL & $12.4(11.5-13.4)$ & $12.8(11.9-14.0)$ & $12.5(11.7-13.6)$ & 0.746 \\
\hline \multicolumn{5}{|l|}{ Inflammatory markers } \\
\hline Erythrocyte sedimentation rate, $\mathrm{mm} / \mathrm{hr}$ & $51(23-71)$ & $50(35-70)$ & $50(31-70)$ & 0.816 \\
\hline C-reactive protein, $\mathrm{mg} / \mathrm{L}$ & $0.8(0.2-4.7)$ & $3.8(0.5-11.7)$ & $1.5(0.2-7.2)$ & 0.008 \\
\hline \multicolumn{5}{|l|}{ Biochemical tests } \\
\hline Blood urea nitrogen, mg/dL & $13(11-17)$ & $14(12-19)$ & $14(11-18)$ & 0.105 \\
\hline Creatinine, $\mathrm{mg} / \mathrm{dL}$ & $0.7(0.6-0.9)$ & $0.8(0.6-1.0)$ & $0.7(0.6-0.9)$ & 0.956 \\
\hline eGFR, mL/min/1.73 m² & $92(77-109)$ & $89(73-107)$ & $91(75-108)$ & 0.040 \\
\hline Sodium, $\mathrm{mmol} / \mathrm{L}$ & $140(138-142)$ & $140(136-142)$ & $140(137-142)$ & 0.070 \\
\hline Potassium, mmol/L & $4.2(3.9-4.5)$ & $4.1(3.7-4.4)$ & $4.1(3.8-4.4)$ & 0.333 \\
\hline
\end{tabular}

Data are presented as the number (\%) or median (interquartile range).

COVID-19, coronavirus disease 2019; ALT, alanine aminotransferase; ULN, upper limit of normal; AST, aspartate aminotransferase; ALP, alkaline phosphatase; GGT, gamma glutamyl transferase; eGFR, estimated glomerular filtration rate.

\section{Clinical outcomes and overall prognosis}

Comparisons of clinical outcomes and prognosis between COVID-19 patients with and without liver enzyme abnormalities are summarized in Table 4. During hospitalization, complications including ARDS, acute kidney injury, and septic shock occurred more frequently in patients with abnormal aminotransferases (ARDS, $20.7 \%$ vs $4.3 \%$; acute kidney injury, $9.4 \%$ vs $2.0 \%$; septic shock, $16.0 \%$ vs
3.3.\%; all values of $\mathrm{p}<0.001)$. A higher proportion of patients with abnormal liver enzymes received oxygen support, including nasal cannulas ( $22.9 \%$ vs $15.6 \%, \mathrm{p}=0.006)$, high-flow nasal cannulas ( $7.2 \%$ vs $2.1 \%, \mathrm{p}<0.001)$, invasive mechanical ventilation ( $15.5 \%$ vs $1.4 \%, \mathrm{p}<0.001)$, and extracorporeal membrane oxygenation $(5.0 \%$ vs $0 \%$, $\mathrm{p}<0.001)$.

As of April 30, 2020, the proportion of patients released 
Table 3. Univariate and Multivariate Logistic Regression Analyses of Clinical Factors Associated with Elevated Aminotransferase Levels in COVID-19 Patients

\begin{tabular}{|c|c|c|c|}
\hline \multirow{2}{*}{ Variable } & \multirow{2}{*}{$\begin{array}{c}\text { Univariate analysis } \\
\text { p-value }\end{array}$} & \multicolumn{2}{|c|}{ Multivariate analysis } \\
\hline & & p-value & Adjusted OR (95\% Cl) \\
\hline Age & 0.469 & & \\
\hline Male sex & 0.001 & 0.035 & $1.500(1.029-2.184)$ \\
\hline Body mass index & $<0.001$ & 0.024 & $1.097(1.012-1.189)$ \\
\hline \multicolumn{4}{|l|}{ Comorbidities } \\
\hline Chronic liver disease & 0.675 & & \\
\hline Liver cirrhosis & 0.311 & & \\
\hline \multicolumn{4}{|l|}{ Signs and symptoms on admission } \\
\hline Fever/chills & 0.001 & & \\
\hline Dyspnea & $<0.001$ & & \\
\hline SIRS on admission & $<0.001$ & & \\
\hline Severe disease of COVID-19 on admission & $<0.001$ & 0.001 & $2.377(1.458-3.875)$ \\
\hline \multicolumn{4}{|l|}{ Radiologic findings on admission } \\
\hline Abnormal chest radiographs & 0.001 & & \\
\hline Bilateral involvement of chest radiographs & 0.001 & & \\
\hline \multicolumn{4}{|l|}{ Laboratory findings on admission } \\
\hline White blood cell count, $/ \mu \mathrm{L}$ & 0.001 & & \\
\hline Platelet count, $/ \mu \mathrm{L}$ & $<0.001$ & $<0.001$ & $0.995(0.993-0.998)$ \\
\hline C-reactive protein, $\mathrm{mg} / \mathrm{L}$ & 0.453 & & \\
\hline
\end{tabular}

Elevated aminotransferase levels are defined as aspartate aminotransferase and/or alanine aminotransferase over twice the upper limit of normal.

COVID-19, coronavirus disease 2019; OR, odds ratio; Cl, confidence interval; SIRS, systemic inflammatory response syndrome.

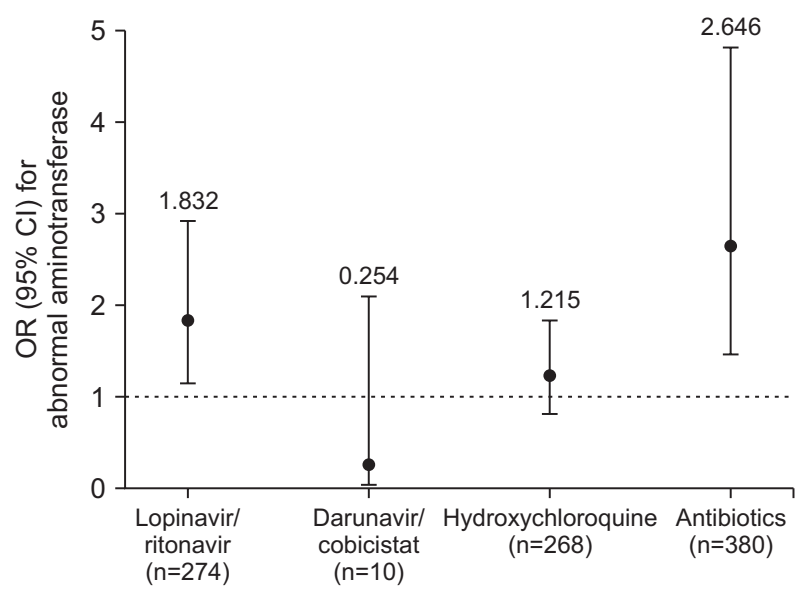

Fig. 1. Adjusted OR $(95 \% \mathrm{Cl})$ for abnormal aminotransferase levels associated with the use of drugs in COVID-19 patients. All results were adjusted for age, sex, underlying liver disease, COVID-19 severity, systemic inflammatory response syndrome, and radiographic findings on admission; $\mathrm{p}=0.011$ for lopinavir/ritonavir, $\mathrm{p}<0.001$ for antibiotics; all p-values $>0.05$ for other drugs.

OR, odds ratio; $\mathrm{Cl}$, confidence interval; COVID-19, coronavirus disease 2019.

from quarantine was higher in the normal than in the abnormal aminotransferase group ( $85.4 \%$ vs $75.4 \%$, $\mathrm{p}<0.001)$. The median time to release from quarantine was significantly shorter in patients with normal aminotransferase than in those with abnormal aminotransferase (22 days vs 26 days, $\mathrm{p}<0.001)$. The overall mortality rate in patients in- cluded in this study was $7.1 \%$. The mortality rate was significantly higher in patients with abnormal liver enzymes than those with normal liver enzymes ( $13.0 \%$ vs $2.9 \%$, $\mathrm{p}<0.001$ ).

\section{DISCUSSION}

In this multicenter retrospective cohort study, the association of clinical features and prognosis of COVID-19 patients with abnormal liver enzymes was evaluated in 874 patients diagnosed with COVID-19 in the Daegu and Gyeongsangbuk-do regions. There were 362 (41.4\%) patients that presented with abnormal aminotransferase, including 94 out of 130 (72.3\%) severe COVID-19 patients and 268 out of 744 (36.0\%) non-severe COVID-19 patients. Male sex, higher body mass index, clinical severity of COVID-19, and lower platelet count on admission emerged as the independent risk factors for elevated aminotransferase levels to more than twice the ULN. The use of lopinavir/ritonavir and antibiotics led to an increased OR of abnormal aminotransferase after admission. Further, abnormal liver enzymes were associated with prolonged time to release from quarantine and higher mortality rates in patients with COVID-19.

Recent studies have reported an incidence of liver damage ranging from $14.8 \%$ to $41 \%$, predominantly charac- 
Table 4. Clinical Outcomes of Patients with COVID-19 According to Liver Test Results

\begin{tabular}{|c|c|c|c|c|}
\hline \multirow{2}{*}{ Outcome } & \multicolumn{2}{|c|}{ Liver tests (aminotransferase) } & \multirow{2}{*}{ Total } & \multirow{2}{*}{$\mathrm{p}$-value } \\
\hline & Normal & Abnormal & & \\
\hline No. of patients & $512(58.6)$ & $362(41.4)$ & 874 & \\
\hline \multicolumn{5}{|l|}{ Complications } \\
\hline Acute respiratory distress syndrome & $22(4.3)$ & $75(20.7)$ & $97(11.1)$ & $<0.001$ \\
\hline Acute kidney injury & $10(2.0)$ & $34(9.4)$ & $44(5.0)$ & $<0.001$ \\
\hline Septic shock & $17(3.3)$ & $58(16.0)$ & $75(8.6)$ & $<0.001$ \\
\hline Continuous renal replacement therapy & $1(0.2)$ & $18(5.0)$ & $19(2.2)$ & $<0.001$ \\
\hline \multicolumn{5}{|l|}{ Oxygen support } \\
\hline Nasal cannula & $80(15.6)$ & $83(22.9)$ & $163(18.6)$ & 0.006 \\
\hline High-flow nasal cannula & $11(2.1)$ & $26(7.2)$ & $37(4.2)$ & $<0.001$ \\
\hline Invasive mechanical ventilation & $7(1.4)$ & 56 (15.5) & $63(7.2)$ & $<0.001$ \\
\hline Extracorporeal membrane oxygenation & 0 & $18(5.0)$ & $18(2.1)$ & $<0.001$ \\
\hline \multicolumn{5}{|l|}{ Prognosis } \\
\hline Release from quarantine & $437(85.4)$ & $273(75.4)$ & $710(81.2)$ & $<0.001$ \\
\hline Hospitalization & $30(5.9)$ & $28(7.7)$ & $58(6.6)$ & 0.273 \\
\hline Transfer to life treatment centers & $30(5.6)$ & $16(4.7)$ & $46(5.3)$ & 0.348 \\
\hline Death & $15(2.9)$ & $47(13.0)$ & $62(7.1)$ & $<0.001$ \\
\hline Time to release from quarantine, day & $22(17-30)$ & $26(19-33)$ & $24(18-31)$ & 0.001 \\
\hline
\end{tabular}

Data are presented as the number (\%) or median (interquartile range). COVID-19, coronavirus disease 2019.

terized by abnormal levels of AST and ALT, occasionally accompanied by elevated ALP levels, in COVID-19 patients. ${ }^{7,11,12}$ Liver synthetic dysfunction has been reported in COVID-19 patients with abnormal liver enzymes, suggesting an association between this impairment and elevated liver enzymes in these patients. ${ }^{7,13}$ Our findings were similar to those previously reported, where $41.4 \%$ of the patients presented with abnormal aminotransferase levels. In contrast, only 50 of 874 patients (6.0\%) presented with elevated ALP levels. In addition, low serum albumin and prolonged international normalized ratio was also observed in patients with abnormal aminotransferases. Thus, the observations from the previous reports and the present study suggest that patients with elevated aminotransferase levels are more likely to have liver synthetic dysfunction.

Angiotensin-converting enzyme II (ACE2), the cell entry receptor of SARS-CoV-2, is highly expressed in bile duct cells. ${ }^{14,15}$ This suggests that SARS-CoV-2 may infect cholangiocytes via ACE2 and cause liver damage. However, ALP (a bile duct injury marker), is not specific to liver disease $^{16}$ or COVID-19., A recent study reported up-regulated ACE2 expression in regenerated liver tissue after acute liver injury in a murine model, suggesting that the compensatory proliferation of hepatocytes expressing ACE2 derived from cholangiocytes may underpin SARS-CoV-2 infection-induced hepatocyte injury. ${ }^{17}$ Abundant SARS-CoV-2 viral particles have been detected in hepatocytes of severe COVID-19 patients with elevated liver enzymes, implying that SARS-CoV-2 infection in hepatocytes induces hepatic impairment in COVID-19 patients. ${ }^{7}$ Although further studies are needed to elucidate the mechanisms of SARSCoV-2-induced liver injury, abnormal liver enzymes are more likely to be due to hepatocyte rather than bile duct damage.

Several recent studies have focused on the prevalence of preexisting liver disease in COVID-19 patients and how it influences the clinical course of COVID-19. A meta-analysis of 73 studies involving 24,299 patients with COVID-19 showed an overall prevalence of chronic liver disease of $3.0 \%,{ }^{18}$ similar to our study (5.0\%). This suggests that patients with preexisting liver disease are not at increased risk of SARS-CoV-2 infection. Several multicenter cohort studies have shown that cirrhosis is associated with higher overall mortality and severe disease course in patients with COVID-19. ${ }^{19-22}$ However, there are little data on whether COVID-19 increases mortality and acute decompensation rates in patients with cirrhosis.

Previous studies on the clinical features of COVID-19 patients showed that abnormal liver enzymes are not associated with specific comorbidities, including hypertension, diabetes, and coronary artery disease. ${ }^{12,23}$ With regard to preexisting liver disease, aminotransferase level abnormalities are present in similar rates among patients with and without chronic liver disease. ${ }^{11,24}$ In our study, patients with abnormal liver enzymes exhibited higher rates of coronary artery disease, but not preexisting liver disease. However, the multivariate analysis did not show a significant association between coronary artery disease and abnormal aminotransferase levels. Definitive evidence on the association between comorbidities and abnormal liver enzyme is still 
lacking.

Male sex, higher body mass index, severe COVID-19 on admission, and lower platelet count were independently associated with elevation of aminotransferase levels to more than twice the ULN in COVID-19 patients. Previous studies have reported that abnormal liver enzymes are more common in males, but the underlying mechanism remains unclear. ${ }^{7,11,12,25}$ Several studies have identified obesity as a significant risk factor for severe COVID- $19^{26,27}$ and that is associated with liver injury (defined as AST and/or ALT over three times the ULN). ${ }^{25}$ An association between liver enzyme abnormalities and severity of COVID-19 was currently reported. ${ }^{7,25} \mathrm{~A}$ recent meta-analysis reported that the platelet count was significantly lower in patients with more severe COVID-19. ${ }^{28}$ Possible causes of liver enzyme abnormalities in severe COVID-19 patients have been proposed, including direct cytotoxicity of SARS-CoV-2, inflammatory cytokine storm, and hypoxic hepatitis. Inflammatory cytokine storm is an excessive or uncontrolled release of proinflammatory cytokines, which exacerbates inflammation and cell injury, finally leading to organ damage. During SARS-CoV-2 infection, an excessive release of proinflammatory cytokines is associated with disease severity. ${ }^{29}$ Recent studies showed that elevated liver enzymes in COVID-19 patients are correlated with higher inflammatory markers. ${ }^{11,13}$ Hypoxic hepatitis can be induced by hemodynamic changes and decreases in oxygen delivery. ${ }^{30}$ Complications, including ARDS, septic shock, and acute cardiac failure, occur mostly in severe COVID-19 patients. ${ }^{3,31}$ These can lead to hepatic artery hypoperfusion and hepatocellular hypoxia, and eventually may cause hypoxic hepatitis. Considering the profound multi-systemic involvement in COVID-19, particularly in patients with severe disease, the elevation of aminotransferase is likely to be multifactorial, involving contributions from the aforementioned factors.

Although effective treatment guidelines for COVID-19 are currently unavailable, antibiotics and antivirals are widely used. Hydroxychloroquine has been used to treat COVID-19 due to its anti-inflammatory and antiviral properties. ${ }^{32-34}$ These drugs may be hepatotoxic in COVID-19 patients. Indeed, recent studies reported that elevated liver enzymes in COVID-19 patients may be due to the use of lopinavir/ritonavir. ${ }^{11,25}$ Our study showed consistent results, indicating that lopinavir/ritonavir use was associated with higher OR of abnormal aminotransferase. To date, data supporting the use of lopinavir/ritonavir to treat COVID-19 are inconclusive, ${ }^{35,36}$ and these drugs should be administered with caution. In our study, the use of antibiotics also increased the OR of abnormal aminotransferase. An association between antibiotic use and liver injury in
COVID-19 patients has been reported. ${ }^{25}$ In consideration of these findings, antibiotics should also be administered with caution for the treatment of SARS-CoV-2-related pneumonia. Clinicians should recognize these issues and carefully monitor liver function tests in COVID-19 patients treated with these drugs.

Compared to patients with normal aminotransferases, those with abnormal aminotransferases had significantly prolonged time to release from quarantine and higher mortality rate, corroborating previous findings. ${ }^{11,12}$ During hospitalization, complications including ARDS, acute kidney injury, and septic shock occurred more frequently in patients with elevated levels of liver enzymes. These complications may have contributed to higher mortality rates in COVID-19 patients with abnormal aminotransferases. Similar to our study, previous studies also reported that the abnormal liver function was associated with prolonged time to release from quarantine in COVID-19 patients. ${ }^{11,12}$ It is possible to speculate that SARS-CoV-2 may persist longer in patients with a higher viral load and lead to hepatocyte damage, which should be assessed in future studies.

Our study has several limitations. First, it was a retrospective study, and there were cases of incomplete medical records of clinical symptoms, laboratory test results, and medical history. Second, data on drugs used as selfmedication before admission were not available. However, most patients were admitted to the hospital within 6 days of symptom onset and rarely used antiviral drugs or antibiotics for self-treatment because these drugs were not available over the counter. Thus, their influence on the study's results is likely to be minimal. Third, we did not use the definition of drug-induced liver injury proposed by the clinical practice guidelines of the European Association for the Study of the Liver, although we demonstrated that abnormal aminotransferase was associated with lopinavir/ ritonavir and antibiotic use. Furthermore, we could not prove causality between these drugs and abnormal aminotransferase using the Roussel Uclaf Causality Assessment Method.

In conclusion, abnormal aminotransferase levels are common in COVID-19 patients and is associated with disease severity. Prolonged time to release from quarantine and higher mortality rates are related to abnormal liver enzymes. Multivariate logistic regression of patients with normal aminotransferase levels on admission showed that the use of lopinavir/ritonavir and antibiotics is associated with abnormal aminotransferase, emphasizing the need for careful monitoring. These results provide clinical guidance for the management of COVID-19 patients during the current pandemic. 


\section{CONFLICTS OF INTEREST}

No potential conflict of interest relevant to this article was reported.

\section{AUTHOR CONTRIBUTIONS}

Study concept and design: B.S.K., W.J.C. Data acquisition: J.E.S., C.H.L., M.K.K., J.G.P., J.S.H., B.K.J., W.Y.J., Y.O.K., W.Y.T., S.Y.P., S.Y.J., Y.R.L. Data analysis and interpretation: J.E.S., B.S.K. Drafting of the manuscript; critical revision of the manuscript for important intellectual content: J.E.S., B.S.K., W.J.C., J.I.S. Statistical analysis: J.E.S., B.S.K. Administrative, technical, or material support; study supervision: B.S.K., W.J.C.

\section{ORCID}

Jeong Eun Song https://orcid.org/0000-0002-1034-4478 Min Kyu Kang https://orcid.org/0000-0002-1435-3312 Yu Rim Lee https://orcid.org/0000-0003-1916-1448 Chang Hyeong Lee

https://orcid.org/0000-0001-5726-8890 Jung Gil Park https://orcid.org/0000-0001-5472-4731 Young Oh Kweon https://orcid.org/0000-0001-5708-7985 Won Young Tak https://orcid.org/0000-0002-1914-5141 Soo Young Park https://orcid.org/0000-0002-4944-4396 Se Young Jang https://orcid.org/0000-0001-9148-9670 Jae Seok Hwang https://orcid.org/0000-0002-1510-3534 Byoung Kuk Jang https://orcid.org/0000-0002-8950-0866 Won Young Jang https://orcid.org/0000-0001-8137-2313 Jeong Ill Suh https://orcid.org/0000-0002-3040-8766 Woo Jin Chung https://orcid.org/0000-0002-3736-1067 Byung Seok Kim https://orcid.org/0000-0002-4318-4570

\section{REFERENCES}

1. Jang JG, Hur J, Hong KS, Lee W, Ahn JH. Prognostic accuracy of the SIRS, qSOFA, and NEWS for early detection of clinical deterioration in SARS-CoV-2 infected patients. J Korean Med Sci 2020;35:e234.

2. Tian S, Hu N, Lou J, et al. Characteristics of COVID-19 infection in Beijing. J Infect 2020;80:401-406.

3. Huang C, Wang Y, Li X, et al. Clinical features of patients infected with 2019 novel coronavirus in Wuhan, China. Lancet 2020;395:497-506.

4. Wang D, Hu B, Hu C, et al. Clinical characteristics of 138 hospitalized patients with 2019 novel coronavirus-infected pneumonia in Wuhan, China. JAMA 2020;323:1061-1069.

5. Chen N, Zhou M, Dong X, et al. Epidemiological and clinical characteristics of 99 cases of 2019 novel coronavirus pneumonia in Wuhan, China: a descriptive study. Lancet 2020;395:507-513.

6. Guan WJ, Ni ZY, Hu Y, et al. Clinical characteristics of coronavirus disease 2019 in China. N Engl J Med 2020;382:17081720.

7. Wang Y, Liu S, Liu H, et al. SARS-CoV-2 infection of the liver directly contributes to hepatic impairment in patients with COVID-19. J Hepatol 2020;73:807-816.

8. Korea Disease Control and Prevention Agency (KDCA). Patient treatment \& management [Internet]. Cheongju: KDCA; c2020 [cited $2020 \mathrm{Feb} 25]$. Available from: http://ncov.mohw. go.kr/en/baroView.do?brdId=11\&brdGubun=112\&dataGub un $=\&$ ncvContSeq $=\&$ contSeq=\&board $\_$id $=\&$ gubun $=$.

9. Shim JJ, Kim JW, Oh CH, et al. Serum alanine aminotransferase level and liver-related mortality in patients with chronic hepatitis B: a large national cohort study. Liver Int 2018;38:1751-1759.

10. World Health Organization (WHO). Laboratory testing for coronavirus disease 2019 (COVID-19) in suspected human cases: interim guidance, 2 March 2020 [Internet]. Geneva: WHO; c2020 [cited $2021 \mathrm{Feb} 2$ ]. Available from: https:// apps.who.int/iris/handle/10665/331329.

11. Fan Z, Chen L, Li J, et al. Clinical features of COVID19-related liver functional abnormality. Clin Gastroenterol Hepatol 2020;18:1561-1566.

12. Xie H, Zhao J, Lian N, Lin S, Xie Q, Zhuo H. Clinical characteristics of non-ICU hospitalized patients with coronavirus disease 2019 and liver injury: a retrospective study. Liver Int 2020;40:1321-1326.

13. Zhang Y, Zheng L, Liu L, Zhao M, Xiao J, Zhao Q. Liver impairment in COVID-19 patients: a retrospective analysis of 115 cases from a single centre in Wuhan city, China. Liver Int 2020;40:2095-2103.

14. Chai X, Hu L, Zhang Y, et al. Specific ACE2 expression in cholangiocytes may cause liver damage after 2019-nCoV infection. bioRxiv [Preprint]. 2020 [cited 2021 Feb 4]. Available from: https://doi.org/10.1101/2020.02.03.931766.

15. Hoffmann M, Kleine-Weber H, Schroeder S, et al. SARSCoV-2 cell entry depends on ACE2 and TMPRSS2 and is blocked by a clinically proven protease inhibitor. Cell 2020;181:271-280.

16. Capelli A, Lusuardi M, Cerutti CG, Donner CF. Lung alkaline phosphatase as a marker of fibrosis in chronic interstitial disorders. Am J Respir Crit Care Med 1997;155:249-253.

17. Guan GW, Gao L, Wang JW, et al. Exploring the mechanism of liver enzyme abnormalities in patients with novel coronavirus-infected pneumonia. Zhonghua Gan Zang Bing Za Zhi 2020;28:100-106. 
18. Kovalic AJ, Satapathy SK, Thuluvath PJ. Prevalence of chronic liver disease in patients with COVID-19 and their clinical outcomes: a systematic review and meta-analysis. Hepatol Int 2020;14:612-620.

19. Hashemi N, Viveiros K, Redd WD, et al. Impact of chronic liver disease on outcomes of hospitalized patients with COVID-19: a multicentre United States experience. Liver Int 2020;40:2515-2521.

20. Kim D, Adeniji N, Latt N, et al. Predictors of outcomes of COVID-19 in patients with chronic liver disease: US multicenter study. Clin Gastroenterol Hepatol 2021;19:1469-1479.

21. Lee YR, Kang MK, Song JE, et al. Clinical outcomes of coronavirus disease 2019 in patients with pre-existing liver diseases: a multicenter study in South Korea. Clin Mol Hepatol 2020;26:562-576.

22. Qi X, Liu Y, Wang J, et al. Clinical course and risk factors for mortality of COVID-19 patients with pre-existing cirrhosis: a multicentre cohort study. Gut 2021;70:433-436.

23. Bloom PP, Meyerowitz EA, Reinus Z, et al. Liver biochemistries in hospitalized patients with COVID-19. Hepatology 2021;73:890-900.

24. Singh S, Khan A. Clinical characteristics and outcomes of coronavirus disease 2019 among patients with preexisting liver disease in the United States: a multicenter research network study. Gastroenterology 2020;159:768-771.

25. Cai Q, Huang D, Yu H, et al. COVID-19: abnormal liver function tests. J Hepatol 2020;73:566-574.

26. Cai Q, Chen F, Wang T, et al. Obesity and COVID-19 severity in a designated hospital in Shenzhen, China. Diabetes Care 2020;43:1392-1398.

27. Docherty AB, Harrison EM, Green CA, et al. Features of 20133 UK patients in hospital with covid-19 using the
ISARIC WHO Clinical Characterisation Protocol: prospective observational cohort study. BMJ 2020;369:m1985.

28. Lippi G, Plebani M, Henry BM. Thrombocytopenia is associated with severe coronavirus disease 2019 (COVID-19) infections: a meta-analysis. Clin Chim Acta 2020;506:145148.

29. Liu J, Li S, Liu J, et al. Longitudinal characteristics of lymphocyte responses and cytokine profiles in the peripheral blood of SARS-CoV-2 infected patients. EBioMedicine 2020;55:102763.

30. Cheung A, Flamm S. Hepatobiliary complications in critically ill patients. Clin Liver Dis 2019;23:221-232.

31. Goha A, Mezue K, Edwards P, Nunura F, Baugh D, Madu E. COVID-19 and the heart: an update for clinicians. Clin Cardiol 2020;43:1216-1222.

32. Biot C, Daher W, Chavain N, et al. Design and synthesis of hydroxyferroquine derivatives with antimalarial and antiviral activities. J Med Chem 2006;49:2845-2849.

33. Devaux CA, Rolain JM, Colson P, Raoult D. New insights on the antiviral effects of chloroquine against coronavirus: what to expect for COVID-19? Int J Antimicrob Agents 2020;55:105938.

34. Fantini J, Di Scala C, Chahinian H, Yahi N. Structural and molecular modelling studies reveal a new mechanism of action of chloroquine and hydroxychloroquine against SARSCoV-2 infection. Int J Antimicrob Agents 2020;55:105960.

35. Cao B, Wang Y, Wen D, et al. A trial of lopinavir-ritonavir in adults hospitalized with severe COVID-19. N Engl J Med 2020;382:1787-1799.

36. Osborne V, Davies M, Lane S, et al. Lopinavir-ritonavir in the treatment of COVID-19: a dynamic systematic benefitrisk assessment. Drug Saf 2020;43:809-821. 